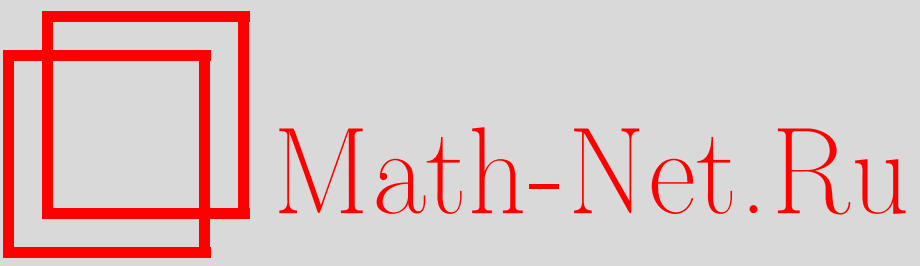

С. Н. Лакаев, Ж. И. Абдуллаев, Спектр четырехчастичного оператора Шрёдингера с парными контактными взаимодействиями на решетке, УМH, 1998, том 53, выпуск 3, 201-202

DOI: https://doi.org/10.4213/rm50

Использование Общероссийского математического портала Math-Net.Ru подразумевает, что вы прочитали и согласны с пользовательским соглашением

http: //www . mathnet.ru/rus/agreement

Параметры загрузки:

IP: 54.162 .27 .143

26 апреля 2023 г., 15:54:12 


\title{
СПЕКТР ЧЕТЫРЕХЧАСТИЧНОГО ОПЕРАТОРА ШРЁДИНГЕРА С ПАРНЫМИ КОНТАКТНЫМИ ВЗАИМОДЕЙСТВИЯМИ НА РЕШЕТКЕ
}

\author{
С. Н. ЛАКАЕВ, Ж. И. АБДУЛЛАЕВ
}

Спектральные свойства многочастичных операторов Шрёдингера на евклидовом пространстве достаточно хорошо изучены [1]-[3]. В частности, установлены конечность или бесконечность числа собственных значений вне существенного спектра этого оператора. При доказательстве конечности или бесконечности дискретного спектра решающим является получение для резольвенты трехчастичного оператора Шрёдингера фредгольмовской системы интегральных уравнений типа Фаддеева или Вайнберга [1]-[3]. Для резольвенты гамильтониана системы четырех квантовых частиц на евклидовом пространстве фредгольмовская система интегральных уравнений типа Фаддеева получена Фаддеевьм и Якубовским [2], [4]. Компактное интегральное уравнение типа Фаддеева для резольвенты гамильтониана системы трех квантовых частиц на решетке получено в работах [5]-[7].

В этом сообщении описан гамилтониан системы четырех одинаковых квантовых частиц на решетке как ограниченньй самосопряженньй оператор (дискретный оператор Шрёдингера ДОШ), действующий в гильбертовом пространстве, получено компактное интегральное уравнение типа Фаддеева-Якубовского для резольвенты ДОШ. Изучена структура существенного спектра четырехчастичного ДОШ и установлено отсутствие собственных значений правее его существенного спектра.

Пусть $T^{\nu}-\nu$-мерньй тор, $L_{2}\left(\left(T^{\nu}\right)^{m}\right)$ - гильбертово пространство всех квадратично интегрируемых функций, определенных на $\left(T^{\nu}\right)^{m}, L_{2}^{\mathrm{sym}}\left(\left(T^{\nu}\right)^{m}\right)$ - подпространство пространства $L_{2}\left(\left(T^{\nu}\right)^{m}\right)$, состоящее из функций $f\left(k_{1}, k_{2}, \ldots, k_{m}\right)$, симметричных относительно перестановки любых двух переменных. В импульсном представлении ДОШ системы четырех одинаковых квантовых частиц действует в $L_{2}^{\mathrm{sym}}\left(\left(T^{\nu}\right)^{4}\right)$ по формуле $F_{\mu} f=F_{0} f+\sum_{1 \leqslant i<j \leqslant 4} V_{i j} f, f \in$ $L_{2}^{\text {sym }}\left(\left(T^{\nu}\right)^{4}\right)$. Здесь $\left(F_{0} f\right)\left(k_{1}, k_{2}, k_{3}, k_{4}\right)=\sum_{i=1}^{4} \varepsilon\left(k_{i}\right) f\left(k_{1}, k_{2}, k_{3}, k_{4}\right)$, а функция $\varepsilon$ является аналитической в некоторой комплексной окрестности тора $T^{\nu}$ и для любого $k$ удовлетворяет условию $\varepsilon(-k)=\varepsilon(k) ; V_{i j}, 1 \leqslant i<j \leqslant 4,-$ интегральный оператор с обобщенным ядром $\delta\left(k_{\alpha}-k_{\alpha}^{\prime}\right) \delta\left(k_{\beta}-k_{\beta}^{\prime}\right) \delta\left(k_{i}+k_{j}-k_{i}^{\prime}-k_{j}^{\prime}\right)$. В дальнейшем $i, j, \alpha, \beta$ обозначают различные индексы, принимающиезначения $1,2,3,4$, т.е. $\{i, j, \alpha, \beta\}=\{1,2,3,4\}$. Например, $\left(V_{34} f\right)\left(k_{1}, k_{2}, k_{3}, k_{4}\right)=$ $\int_{\left(T^{\nu}\right)^{2}} \delta\left(k_{3}+k_{4}-k_{3}^{\prime}-k_{4}^{\prime}\right) f\left(k_{1}, k_{2}, k_{3}^{\prime}, k_{4}^{\prime}\right) d k_{3}^{\prime} d k_{4}^{\prime}$. На торе $T^{\nu}$ выбрана единичная мера, т.е. $\int_{T^{\nu}} d k=1$.

Пусть $N=k_{1}+k_{2}+k_{3}+k_{4}$ - полньй квазиимпульс системы четырех частиц и $\Gamma_{N}=$ $\left\{\left(k_{1}, k_{2}, k_{3}, k_{4}\right) \in\left(T^{\nu}\right)^{4}: k_{1}+k_{2}+k_{3}+k_{4}=N\right\}$ - многообразия размерности $3 \nu, L_{2}^{\mathrm{sym}}\left(\Gamma_{N}\right)-$ гильбертово пространство всех квадратично интегрируемых симметричных функций, определенных на $\Gamma_{N}$. Если в качестве независимых переменных на многообразии $\Gamma_{N}$ берем любую тройку из переменных $k_{1}, k_{2}, k_{3}, k_{4}$, то элементы пространства $L_{2}^{\text {sym }}\left(\Gamma_{N}\right)$ удовлетворяют условию $f\left(k_{1}, k_{2}, k_{3}\right)=f\left(N-k_{1}-k_{2}-k_{3}, k_{2}, k_{3}\right)$.

Пространство $L_{2}^{\mathrm{sym}}\left(\Gamma_{N}\right)$ и оператор $F_{\mu}$ могут быть представлены в виде прямых интегралов: $L_{2}^{\mathrm{sym}}\left(\left(T^{\nu}\right)^{4}\right)=\int_{T^{\nu}} \oplus L_{2}^{\mathrm{sym}}\left(\Gamma_{N}\right) d N, F_{\mu}=\int_{T^{\nu}} \oplus F_{\mu}(N) d N$, где ограниченный самосопряженный оператор $F_{\mu}(N)$ действует в гилбертовом пространстве $L_{2}^{\mathrm{sym}}\left(\Gamma_{N}\right)$ по формуле:

$$
F_{\mu}(N) f=F_{0}(N) f-\mu \sum_{1 \leqslant i<j \leqslant 4} V_{i j} f, \quad\left(F_{0}(N) f\right)\left(k_{1}, k_{2}, k_{3}\right)=\varepsilon_{N}\left(k_{1}, k_{2}, k_{3}\right) f\left(k_{1}, k_{2}, k_{3}\right),
$$

$f \in L_{2}^{\mathrm{sym}}\left(\Gamma_{N}\right), \varepsilon_{N}\left(k_{1}, k_{2}, k_{3}\right)=\varepsilon\left(k_{1}\right)+\varepsilon\left(k_{2}\right)+\varepsilon\left(k_{3}\right)+\varepsilon\left(N-k_{1}-k_{2}-k_{3}\right)$. В дальнейшем вместо переменного $N-k_{1}-k_{2}-k_{3}$ мы используем обозначение $k_{4}$. Из симметричности функции $f \in L_{2}^{\mathrm{sym}}\left(\Gamma_{N}\right)$ относительно переменных действие оператора $V_{i j}, 1 \leqslant i<j \leqslant 4$, на $f$ можно определить следующим образом: $\left(V_{i j} f\right)\left(k_{1}, k_{2}, k_{3}\right)=\int_{T^{\nu}} f\left(k_{\alpha}, k_{\beta}, s\right) d s=\varphi\left(k_{\alpha}, k_{\beta}\right)$. Например, $\left(V_{12} f\right)\left(k_{1}, k_{2}, k_{3}\right)=\int f\left(k_{3}, k_{4}, s\right) d s=\int f\left(k_{3}, N-k_{1}-k_{2}-k_{3}, s\right) d s$. Пространство

Работа финансируется Фондом поддержки фундаментальных исследований АН РУз. 
$L_{2}^{i j}\left(\Gamma_{N}\right)$ состоит из тех и только тех элементов $f \in L_{2}\left(\Gamma_{N}\right)$, которые симметричны относительно переменных $k_{i}, k_{j}$ и $k_{\alpha}, k_{\beta}$. Введем следуюшие операторы каналов:

$$
\begin{aligned}
h_{\mu}^{i j}(N) f & =F_{0}(N) f-\mu V_{i j} f, & & f \in L_{2}^{i j}\left(\Gamma_{N}\right), \\
h_{\mu}^{i j, \alpha \beta}(N) f & =F_{0}(N) f-\mu\left[V_{i j}+V_{\alpha \beta}\right] f, & & f \in L_{2}^{i j}\left(\Gamma_{N}\right), \\
H_{\mu}^{i j \alpha}(N) f & =F_{0}(N) f-\mu\left[V_{i j}+V_{i \alpha}+V_{j \alpha}\right] f, & & f \in L_{2}^{s}\left(\Gamma_{N}\right) .
\end{aligned}
$$

Операторы каналов разлагаются на прямые интегралы:

$$
\begin{aligned}
h_{\mu}^{i j}(N) & =\int_{T^{\nu}} \oplus\left[h_{\mu}(k) \otimes I+I \otimes h_{0}(N-k)\right] d k, \\
h_{\mu}^{i j, \alpha \beta}(N) & =\int_{T^{\nu}} \oplus\left[h_{\mu}(k) \otimes I+I \otimes h_{\mu}(N-k)\right] d k, \\
H_{\mu}^{i j \alpha}(N) & =\int_{T^{\nu}} \oplus\left[H_{\mu}(K)+\varepsilon(N-K) E\right] d K .
\end{aligned}
$$

Здесь $h_{\mu}(k), k=k_{i}+k_{j},-$ двухчастичный оператор Шрёдингера, $H_{\mu}(K), K=k_{i}+k_{j}+k_{\alpha}$, - трехчастичньй оператор Шрёдингера, определяемые, соответственно, по формулам

$$
\begin{gathered}
\left(h_{\mu}(k) f\right)(p)=[\varepsilon(k-p)+\varepsilon(p)] f(p)-\mu \int_{T^{\nu}} f(s) d s, \\
\left(H_{\mu}(K) f\right)(p, q)=\varepsilon_{K}(p, q) f(p, q)-\mu \int_{T^{\nu}}[f(p, s)+f(q, s)+f(K-p-q, s)] d s,
\end{gathered}
$$

где $\varepsilon_{K}(p, q)=\varepsilon(K-p-q)+\varepsilon(p)+\varepsilon(q)$. Операторы $h_{\mu}(k)$ и $H_{\mu}(K)$ действуют, соответственно, в гильбертовых пространствах $\tilde{L}(k)$ и $L(K)$. Здесь $\tilde{L}(k)$ - гильбертово пространство, состоящее из функций $f \in L_{2}\left(T^{\nu}\right)$, удовлетворяющих условию $f(p)=f(k-p)$, а $L(K)$ - подпространство пространства $L_{2}^{\mathrm{sym}}\left(\left(T^{\nu}\right)^{2}\right)$, элементы которого удовлетворяют условию $f(p, q)=$ $f(p, K-p-q), I$ и $E$ - единичные операторы в пространствах $\tilde{L}(k)$ и $L(K)$, соответственно. Знак $\otimes$ используется для тензорного произведения операторов.

Tеорема 1. Существенный спектр $\sigma_{\mathrm{ess}}\left(F_{\mu}(N)\right)$ оператора $F_{\mu}(N)$ совпадает с множеством $\Sigma(N)$, m.е. $\sigma_{\mathrm{ess}}\left(F_{\mu}(N)\right)=\sigma\left(h_{\mu}^{i j}(N)\right) \cup \sigma\left(h_{\mu}^{i j, \alpha \beta}(N)\right) \cup \sigma\left(H_{\mu}^{i j \alpha}(N)\right)=\Sigma(N)$.

ТеОРема 2. Для любого $\mu \in(0, \infty)$ оператор $F_{\mu}(N)$ не имеет собственных значений, лежащих правее его существенного спектра.

Обозначим через $T(z)$ оператор $\left(1-\mu V_{12} R_{0}(z)\right)^{-1} V_{12} R_{0}(z), \quad T(z) V_{i j} f=T_{i j}(z) \varphi$, $I+\mu R_{i j}(z)$ - резольвента оператора $I-\mu T_{i j}(z)$. Определим оператор $R_{\mu, N}(z)$, действующий в гильбертовом пространстве $L_{2}^{s}\left(\left(T^{\nu}\right)^{2}\right)$, по формуле

$$
\begin{aligned}
R_{\mu, N}(z) \varphi=2 \mu^{2}[ & R_{34}(z) T_{23}(z)+R_{34}(z) T_{13}(z)+R_{13}(z) T_{34}(z) \\
& \left.+2 R_{13}(z) T_{23}(z)+R_{23}(z) T_{34}(z)+2 R_{23}(z) T_{13}(z)\right] \varphi .
\end{aligned}
$$

Теорема 3. Число $z \in \mathbb{C} \backslash \sigma_{\mathrm{ess}}\left(F_{\mu}(N)\right)$ является собственным значением оператора $F_{\mu}(N)$ тогда и только тогда, когда оператор $R_{\mu, N}(z)$ имеет собственное значение, равное единице.

\section{СПИСОК ЛИТЕРАТУРЫ}

[1] Рид М., Саймон Б. Методы современной математической физики. Т. 4. Анализ операторов. М.: Мир, 1982. [2] Меркурьев С.П., Фаддеев Л. Д. Квантовая теория рассеяния для систем несколюких частиц. М.: Мир, 1985. [3] Цикон Х., Фрези Р., Кирш В., Саймон Б. Операторы Шрёдингера. М.: Мир, 1990. [4] Шмидт Э., Цигельман Х. Проблемы трех тел в квантовой механике. М.: Мир, 1979. [5] Mattis D. C. // Rev. Modern Phys. 1986. V. 58. № 2. Р. 361-379. [6] Лакаев С. Н. // ТМФ. 1991. Т. 89. № 1. С. 94-104. [7] Лакаев С. Н. // Функц. анализ и его прил. 1993. Т. 27. № 3. С. 15-28. 